\title{
Las versiones del desarrollo sostenible
}

\author{
Claudia Eugenia Toca Torres \\ Doutora em Ciências Políticas e Sociais (Universidad Nacional Autónoma de México) \\ Professora na Universidad del Rosario \\ Bogotá, Colombia \\ claudia.toca@urosario.edu.co
}

\begin{abstract}
Resumen En el marco del desarrollo sostenible hay una tendencia a vincular el privilegio del medio ambiente con el biocentrismo y la sociedad con el antropocentrismo, sin darse cuenta de la verdadera esencia de éstas versiones y la existencia de otras. Desde el antropocentrismo es válida la satisfacción de intereses básicos y de necesidades vitales del hombre, evitando o reduciendo el daño a la naturaleza. Por su parte, el biocentrismo pretende el reconocimiento del valor intrínseco de las especies, permitiendo el uso de la naturaleza pero no su explotación o la dominación. Si bien el biocentrismo se soporta fundamentalmente en la ética y el antropocentrismo en la política, no se puede negar que las dos versiones tienen amplia relación con las tradiciones ideológicas.
\end{abstract}

Palabras claves: ecocentrismo; antropocentrismo; desarrollo sostenible

\section{Introducción}

$\mathrm{E}$ N El Debate sobre el DeSARrollo sostenible, existen dos visiones opuestas que reflejan una profunda división en las ciencias sociales con respecto al papel y al lugar de la ciencia y el conocimiento en la sociedad moderna. Se trata del debate entre un enfoque determinista y uno más constructivista sobre la ciencia. En la visión determinista, la ciencia habla sobre la verdadera naturaleza del mundo real, de acuerdo con ella, los asuntos de sostenibilidad tienen que ver con nuestra realidad biofísica y los límites impuestos a las actividades humanas (Meadows et al., 1972, Meadows, 1992, Hueting; Reijnders apud Hermans; Knippenberg, 2006, p. 300). De acuerdo con la visión constructivista los problemas de sostenibilidad son socialmente construidos y como resultado de ello son intrínsecamente subjetivos (Hermans; Knippenberg, 2006, p. 300).

Muchas han sido las definiciones del desarrollo sostenible, sin embargo, aquellas que involucran las dimensiones social y ambiental han sido objeto de discusión, especialmente, cuando se trata de determinar cuál de las dos domina. El dilema emergente se centra en el bienestar de la especie humana versus el de las especies naturales (animal y vegetal). En apariencia, el dilema desaparecería al garantizar un equilibrio en las dos dimensiones, no obstante, conviene rastrear las visiones que soportan cada una para comprender la existencia de ideologías y en consecuencia movimientos que impactan agendas locales en materia ambiental y social. Dado que las discusiones sobre las versiones del desarrollo sostenible no están reservadas para los filósofos, en esta ocasión se intentará una revisión de los trabajos de estudiosos de disciplinas diversas. 


\section{Versiones del Desarrollo Sostenible}

Partiendo de la idea que el desarrollo sostenible trata de vincular el desarrollo económico con la calidad ambiental y social, eso conlleva en la combinación del crecimiento ambientalmente sostenible con un enfoque sobre equidad inter e intrageneracional y distribución equitativa de bienestar entre grupos y naciones (McIntosh apud Rao, 1998, p. 65). Aunque para algunos autores, este desarrollo es literal y claramente un sentido ecológico y otro social (Fergus; Rowney, 2005, p. 19), no es raro encontrar el uso de la denominación "sostenible" para referirse exclusivamente a asuntos verdes, ecológicos o ambientalmente amigables. Se identifica, por lo tanto, una preferencia por la dimensión ecológica y una omisión de lo social, surgiendo entonces un interrogante en torno a los orígenes de dichas inclinaciones, respuesta que puede encontrarse en las versiones del desarrollo sostenible.

En torno al desarrollo sostenible han sido reconocidas dos versiones: una fuerte y una débil. La idea clave de la Comisión Mundial del Ambiente y el Desarrollo (1987) de crecer económicamente evitando la degradación ambiental, parece salvar las diferencias entre los fines deseados y los medios indeseados. Desafortunadamente, dichas diferencias sólo pueden superarse en el nivel más abstracto (Eden, 1994, Carvalho apud Kallio, 2007, p. 48). El desarrollo sostenible está tratando de salvar las enormes diferencias entre los objetivos y las interpretaciones débiles y fuertes.

La versión fuerte o ecocéntrica pretende un equilibrio aceptable entre la sociedad y el ecosistema natural. Socialmente, eso implica una reducción de la población humana y una redistribución de la riqueza dando más a los pobres y menos a los ricos. La versión débil o antropocéntrica pone el ecosistema natural al servicio de la sociedad, lo que justifica su uso y agotamiento. En términos sociales significa que el ecosistema natural debe ser sobreexplotado hasta que los pobres tengan la misma riqueza que los ricos. Tan pronto como este punto haya sido logrado, la población mundial en su totalidad podrá trabajar sobre los aspectos ecológicos de sostenibilidad (Klostermann; Cramer, 2006, p. 268-269).

Estas versiones han dado lugar a la caracterización tradicional del desarrollo sostenible: débil y fuerte. Las interpretaciones de debilidad están rodeadas de una visión antropocéntrica del mundo y han expresado, típicamente, la esencia del desarrollo sostenible en términos de propósitos individuales más que sociales. Las demandas por equidad intrageneracional han sido ignoradas y el bienestar de otras especies resulta secundario para el bienestar humano. El ambiente natural es visto como un recurso a ser dominado y agotado. A su vez,, las interpretaciones de fortaleza se niegan a tratar de las especies humana y natural por separado, tratan de mantener las posibilidades de la vida, reconciliando el desarrollo humano y la integridad ambiental. Mientras que las interpretaciones débiles tienden a confiar en estructuras autoritarias y coercitivas, tales como fuerzas de mercado, las interpretaciones fuertes consideran que se requieren procesos más participativos, transparentes y democráticos (Davidson, 2000, Bebbington, 2001, Avres et al. apud Kallio, 2007, p. 42).

El punto fundamental de la discusión entre ecocentrismo y antropocentrismo es la relación del hombre con la tierra, con los animales y con las plantas. La propagación de la ética de los últimos (tierra, animales y plantas) entre la especie humana es "una posibilidad evolutiva y una necesidad ecológica" (Leopold apud Kortenkamp; Moore, 2001, p. 261). Por tiempos, los temas ambientales se consideran verdaderos dilemas de prisionero, pues cada individuo recibe un pago más alto por una decisión socialmente egoísta (contaminar), por una decisión socialmente cooperativa, sin importarse con lo que hacen otros individuos de la sociedad. No ha sido posible hacerles entender que es mejor cooperar que traicionar, cuando se trata de bienestar colectivo (Kortenkamp; Moore, 2001, p. 261). Autores como Stern y Mechant, a partir de Leopold, han identificado tres niveles de interés y que se puede ver en la figura 1: el interés propio, el altruismo humanista o social y el altruismo biosférico o biocentrismo (Dietz; Fitzgerald; Shwom, 2005, p. 344).

El nivel individual es propio del modelo del actor racional, por lo que las decisiones se tomen a partir de interés individual; el altruismo humano, se debe a una amplia zona de preocupación del plan personal, familiar o de una comunidad más amplia, incluyendo, posiblemente, de toda la humanidad. El altruismo biosférico está dirigido hacia otras especies o hacia el estado de estos ecosistemas más allá de los beneficios proporcionados a los humanos. Los dos primeros son antropocéntricos por lo que asignan un valor instrumental a otras especies del ambiente, mientras que la biosfera, es un área de preocupación más allá de los límites del homo sapiens. El altruismo biosférico implica asignar peso a otras especies, ecosistemas e incluso a la propia biosfera (Dietz; Fitzgerald; Shwom, 2005, p. 344-345).

Figura 1 - Niveles de interés

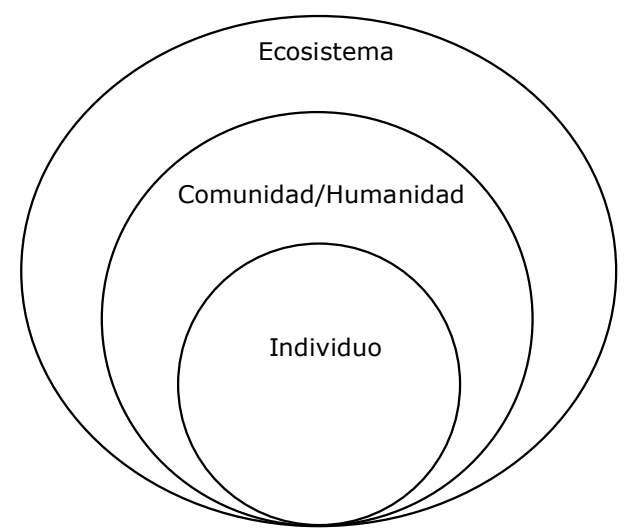

Fuente: Elaboración con información de Dietz; Fitzgerald; Shwom (2005) 
En el marco de las políticas públicas ambientales es posible garantizar la versión débil del desarrollo sostenible y no apenas la fuerte, asimismo, las políticas sociales pueden contener un rasgo ecocéntrico. Un enfoque antropocéntrico de la política ambiental parte de la consideración de los individuos, su análisis incluye métodos basados en la utilidad y en los derechos. La literatura sobre éstos últimos tiende a centrarse en las condiciones ambientales de los grupos marginados (pobres, minorías o indígenas), la literatura sobre la utilidad se centra en los costos y beneficios sociales agregados de la regulación ambiental. Por su parte, un enfoque ecocéntrico de la política ambiental se deriva de la perspectiva del ambiente natural en su conjunto ecológico, como un grupo de especies o entes individuales. Desde esta perspectiva implica enfoques religiosos de ecopolítica en la que debemos proteger el ambiente natural, ya que se trata de un producto divino. Incluye además, conceptos tan complejos de la ecología que, razonablemente, no se puede desagregar en partes los componentes y debe interactuar con ella en su totalidad (Barkin, 2006, p. 57-58). Las tendencias ecocéntricas reconocen como cualidades de la naturaleza el valor, el bien y el mérito, en contrapartida, los enfoques antropocéntricos resisten a la adscripción de dichas cualidades en la naturaleza (Scott, 2003, 64).

\section{Enfoques centrados en la naturaleza}

El ecoradicalismo es un enfoque ambiental centrado en la liberación de todos los humanos y animales del sometimiento, de subordinación y de dominación. Implica un compromiso con la promoción de la comunidad, la unidad y con compartir los recursos más allá de las divisiones de clase de género y raciales. Ejemplos de enfoques ecoradicales incluyen: ecología profunda, biocentrismo (ecocentrismo), ecofeminismo, ecología social, derechos de los animales, ecopsicología, justicia ambiental, política verde, ecofenomenología, bioregionalismo y construcción social de la naturaleza (Esbjörn-Hargens, 2005, p. 26).

Algunos filósofos ambientales han acusado a sus colegas más radicales de promover una especie de ecofascismo, en el que eleva el todo orgánico, es decir de la Tierra, por encima de las necesidades e intereses de los organismos individuales, incluso los animales y los humanos. Estos extremistas ambientales se denominan fascistas por ser románticos amantes de la naturaleza con tendencias reaccionarias. La ecología radical con un enfoque biocéntrico promueve la idea de que la especie humana no es privilegiada, sino un miembro de la compleja comunidad biótica. Un tipo de ecología radical 'ecología profunda' afirma que las consecuencias de la arrogancia humana (expresada en una tecnología industrial desenfrenada y un consumismo inconsciente) están amenazando la integridad de la biosfera (Zimmerman, 1995, p. 208-209).

La ecología profunda es un concepto acuñado por el filósofo noruego Arne Naess, que engloba las visiones de muchos pensadores ambientales, que obtienen sus conclusiones de diferentes maneras. Al mantener los principios de la ecología profunda han emergido otros conceptos como la ecología ecocéntrica y la ecología transpersonal (DiZerega, 1996, p. 700). Naess identificó una posición filosófica propia para la ecología, se trata de la ecofilosofía o como la denominó Ecosofía T, constituida por conceptos como la autorrealización y el valor intrínseco. Bajo esta posición, se distinguen dos tipos de acciones del individuo: las obligatorias que emergen de las leyes morales (ética) y las nobles ejecutadas de manera espontánea y jubilosa como expresión del yo (Curtin, 1999, p. 130). La ecología profunda sostiene que las plantas, los animales, las formas naturales de la tierra, el agua y el aire, merecen respeto independientemente de la utilidad proporcionada a los seres humanos. (DiZerega, 1995, p. 240), siempre ha criticado el antropocentrismo y se ha inclinado hacia el ecocentrismo.

Por su parte, Devall y Sessions argumentan que la tarea de la ecología profunda consiste en reversar tendencias destructivas de la civilización moderna, es decir, ver la naturaleza como un organismo. Asimilar la naturaleza a un organismo, implica un método de estudio holístico más que reduccionista (Kirkman, 2002 , p. 21). Se califica como profunda por explorar supuestos fundamentales de nuestros valores y de la experiencia del mundo, así mismo, porque las actitudes que la respaldan - aunque inspiradas por diversas fuentes - , revelan una dependencia íntima de la humanidad con la justificación racional de los ecologistas sobre conductas decentes con el ambiente natural (Palmer, 2000, p. 212). La ecología profunda no separa los humanos de la naturaleza, no ve el mundo como una colección de objetos aislados, sino como una red de fenómenos que están interconectados y son interdependientes (Hoy, 2000, p. 94).

Naess y Sessions desarrollaron los principios para esta ecología: 1) el bienestar y la prosperidad de la vida en la Tierra (humana y no humana) tienen valor en si mismos - valor intrínseco o inherente; 2) la riqueza y la diversidad de las formas de vida contribuyen a la realización del valor intrínseco, además de constituirse en valores en si mismos; 3) los humanos no tienen derecho a reducir esta riqueza y diversidad, excepto para la satisfacción vital de necesidades humanas; 4) la prosperidad de la vida humana y las culturas es compatible en la medida que se de una disminución sustancial de la población humana; 5) la actual interferencia humana en el mundo no humano es excesiva por lo que la situación se empeora rápidamente; 6) deben cambiarse las políticas pues están afectando las 
estructuras económicas, tecnológicas e ideológicas básicas; 7) el cambio ideológico es lo que aprecia la calidad de vida en lugar de adherirse a estándares de vida más altos; y 8) quienes se suscriban a los anteriores puntos tienen una obligación con la implementación directa o indirecta de los cambios necesarios (DiZerega, 1996, p. 701, Edwards, 2005, p. 115-116).

El valor intrínseco se reconoce como el principio que ha atraído más atención, es decir, aquella visión que considera que el bienestar y la prosperidad de las especies de la Tierra (humanas y no humanas) tienen valor en si mismas. Bajo esta óptica, los ecosistemas y especies salvajes, tienen un valor intrínseco y el derecho de existir, resultando necesarios para la salud ecológica del planeta y para maximizar el bienestar de los humanos (Scott, 2003, 67). A su vez, el principio sobre la riqueza y la diversidad de las formas de vida se ha identificado como igualitarismo biocéntrico (Mathews, 1993, p. 120). Bajo este principio, todos los elementos de la biosfera tienen el mismo derecho a vivir, prosperar y alcanzar sus propias formas de desarrollo y logros. Todos los organismos y entes en la ecosfera como partes de un todo interrelacionado son iguales en cuanto a valor intrínseco (Devall; Sessions apud Mathews, 1993, p. 126).

Como movimiento teórico, el lema de la ecología profunda "primero la Tierra" logró convencer que la conservación del ambiente difícilmente puede alcanzarse por medios políticos usuales (Scott, 2003, p. 66). Entre las políticas recomendadas por el propio Naess figuran: reducción radical de la población del mundo, sacrificio de metas de crecimiento económico en el mundo desarrollado, conservación de la diversidad biótica y desarrollo de comunidades autosostenibles. Como holístico, Naess sostiene que en un nivel fundamental, todos los organismos están intrínsecamente relacionados en una red o campo biosférico (Palmer, 2000 , p. 213). De igual modo, enfatiza la importancia de entender la ecología profunda como una plataforma, dicho de otro modo, su atractivo puede convocar diferentes visiones filosóficas y religiosas en torno a una excusa común (Scott, 2003, p. 68).

La práctica de la ecología profunda implica fomentar una actitud no dualística, tolerante, apreciativa y respetuosa hacia la complejidad e integridad de la ecosfera en su conjunto. Mientras que la posición de los activistas modernos sumerge al género humano en el frenesí del ciclo de producción y de consumo y pone en evidencia la soledad de los seres no humanos en términos instrumentales; la postura de la ecología profunda fomenta la compasión y benevolencia hacia todos los seres, concibe al género humano como un miembro integrante de la comunidad ecológica, invita a las personas a desplazarse del consumismo irracional y en su lugar satisfacer apenas las necesidades materiales vitale (Zimmerman, 2002, p. 41).

John Passmore (1974) propuso una distinción útil entre problemas en ecología esencialmente científicos que pueden orientarse e incluso resolverse a través de la investigación experimental y el cambio tecnológico; y una categoría mucho más amplia de problemas ecológicos que son de carácter cultural y propios de la sociedad derivados de la relación de los humanos con la naturaleza. Por ejemplo, la contaminación, claramente incluye las cuestiones de la ecología: análisis toxicológico, evaluación del impacto ambiental y mejora tecnológica. La definición de lo que es contaminación es un tema cultural que depende tanto del cambio de valores y prioridades como de las emisiones actuales de sustancias tóxicas (Garrard, 2007, 3p. 59).

En el ensayo "Imperialismo y Ambientalismo", Eric Katz formula una serie de interrogantes que hacen pensar en torno a los enfoques ecoradicales ¿Cuándo la intervención se convierte en intento de dominación? De alguna manera, los humanos deben comer; cultivar sus alimentos; construir casas, carreteras, ciudades; y curar sus enfermedades entre otros intereses básicos. ¿Son todos estos actos de dominación imperialista moralmente cuestionables? Es así que para Katz no todas las interacciones son casos de relaciones desiguales de poder algunas son simbióticas. Caben adicionalmente interrogantes del tipo ¿Buscamos los humanos un equilibrio con la naturaleza, un tipo de sociedad o una relación poderosa de control y dominación? Respecto a los procesos agrícolas ¿Son orgánicos o trabajados con procesos naturales? ¿Son altamente tecnificados? ¿Se controlan las plagas mediante fertilizantes y pesticidas artificiales? (Hettinger, 2002, p. 119).

Las posibilidades en función de la naturaleza sin negar que ésta siempre actúe como medio - serán dos usarla o explotarla. El uso siempre tendrá una connotación menos dañina frente a la existencia de las necesidades básicas, debido a que no está desprovisto de respeto e interés por su floración. Por otro lado, el cambio de la naturaleza por el hombre no demanda dar como resultado una realidad puramente artificial carente de valor natural. En caso de ser éste el resultado, evidentemente, se trataría de una dominación de la naturaleza en lugar de una interacción con ella. La intervención humana puede contribuir valorando la naturaleza en términos de su diversidad, belleza, estabilidad y complejidad. Por ejemplo, aunque plantar flores en los jardines, domesticar a los animales y concebir los paisajes rurales sean acciones que reducen la independencia de la naturaleza, es posible verlas como aportantes a su valor en términos de belleza y diversidad. Si es así, la interacción humana con la naturaleza puede ser una relación mutuamente beneficiosa, sin incurrir en una degradación general del valor natural (Hettinger, 2002, p. 120).

Los ecosistemas no subordinan sus componentes a un programa central, son policéntricos y acéntricos (Morín apud Whiteside, 2004, p. 363). El policentrismo se refiere el hecho que a pesar de integrar 
y moldear las actividades de diversas especies, dichos sistemas permiten sus identidades para mantenerse distintos. Son acéntricos en la medida que continuamente se reorganizan, pierden una identidad determinada. Cuanto más alto sea el nivel de complejidad menos aplicable será la idea que un organismo vivo tiene un centro. La orientación normativa del trabajo de Morin deviene del concepto de adaptación a través de eco-reorganización. La meta de la teorización ecológica de Morin consiste en identificar y promover condiciones sociales que proporcionen a la humanidad mejores oportunidades para una transición hacia un nivel de complejidad mayor exitosamente realizada. Se trata de identificar tendencias en un mundo en el que fuentes en favor de la creatividad evolutiva sean adecuadamente fomentadas, fuentes capaces de trascender aspectos ecológicamente disfuncionales de las sociedades modernas (Whiteside, 2004, p. 363).

Las perspectivas ecológicamente profundas han sido descritas como biocéntricas, ecocéntricas o transpersonales (DiZerega, 1995, p. 239). En el marco de una versión ecocéntrica el desarrollo sostenible no es apenas una cuestión ambiental, sino una cuestión más incluyente sobre cómo debería o podría estar organizada la relación hombre - sociedad - naturaleza - empresa, incluyendo, por supuesto, los asuntos de justicia intra e intergeneracional (Kallio, 2007, p. 46). En esta relación, se aprecia que la línea entre humanidad y naturaleza es una construcción social. "Los seres humanos han construido una jerarquía moral asumiendo que ellos están por encima o apartados de otras criaturas más humildes" (Purser et al. apud Kallio, 2007, p. 47). El ecocentrismo invita a las personas a respetar a los seres individuales y al ecosistema en el que se desenvuelve y que se ve como un cuerpo (Zimmerman, 2002, p. 41).

El interés ecocéntrico por el ambiente o ecocentrismo predispone a los individuos a valorar la naturaleza por su propio bien, considerando que merece protección dado su valor intrínseco sin tener en cuenta su utilidad para los humanos. El ecocentrismo es paralelo a la orientación biosférica del valor, los individuos están más dispuestos a proteger el ambiente sin importarse que eso implique su molestia y gastos (Casey; Scott, 2006, p. 58).

El biocentrismo de Paul Taylor (1913) es una perspectiva más radical que combina un enfoque virtuoso con elementos de utilitarismo y ética deontológica. Declara el respeto por la vida como principio, sostiene que los intentos por discriminar distintas formas de vida sobre las bases de un criterio general tales como autonomía o sufrimiento son tan arbitrarios como privilegios tradicionales de la especie humana. La debilidad de esta perspectiva radica en la ausencia de un criterio externo, un supuesto teleológico no dice si un particular fin es bueno o malo (ortodoxia cristiana). Este enfoque tiene que ver con el trato igualitario de los intereses de diversas especies, su método tiene similitudes al de los derechos humanos.

Trata de establecer una jerarquía de intereses basada en la autodefensa y los intereses básicos necesarios para que una especie particular prospere, en la que los intereses no básicos son una categoría residual. Un conflicto entre intereses básicos debe ser resuelto en torno al tema de la igualdad, cuando resulte inevitable debe generar el mínimo daño y un deber de cuidado y restitución (Cheyne; Alder, 2007, p. 186).

Por lo tanto, es interesante reflexionar en torno a las necesidades de la especie humana para con el fin de establecer la supremacía de intereses. No se puede ignorar que el uso de la naturaleza no siempre se da en una misma escala, ello es despejar un bosque para destinarlo a la cultura para la supervivencia alimentaria de una comunidad indígena, no es lo mismo que talarlo para construir un confortable multifamiliar. Sin embargo, desde el biocentrismo, la comunidad está obligada a restituir y reparar el daño ocasionado a la especie vegetal.

Los filósofos biocéntricos reconocen moralmente a todos los seres vivientes, los ecocéntricos trabajan desde una ontología relacional y están interesados en la integridad no sólo de las poblaciones y especies, sino también en comunidades ecológicas más amplias en múltiples niveles de agregación (Eckersley, 2007, p. 306).

Eric Katz (1997) aboga por una filosofía ambiental no antropocéntrica, holista y comunitaria que trata a la naturaleza como un objeto directo de la preocupación moral. La naturaleza es objeto para Katz ya que ella tiene sus propios procesos e historia de desarrollo, independientemente, de la intervención y actividad humana. La autonomía humana y la libertad para perseguir su propia independencia y curso de desarrollo no planeado, otorgan a la naturaleza una demanda moral sobre los humanos que Katz identificó como "el llamado de la naturaleza" (Hettinger, 2002, p. 113).

La caracterización de Katz sobre la relación hombre-naturaleza sugiere que cualquier uso humano de la naturaleza es abusivo. Afirma "cuando los humanos moldean y manipulan el mundo natural para lograr sus propios intereses, para satisfacer sus deseos es una forma de dominación antropocéntrica, opresión y negación de la autonomía de la naturaleza. En torno a la visión de Katz han sido identificadas tendencias como una dicotomía estricta desde la distinción natural-artificial haciendo caso omiso de la posibilidad de los grados de diferencia; y asimilar todas las intenciones y propósitos humanos como intentos antropocéntricos de dominación y control de la naturaleza. Una visión positiva de los humanos en la naturaleza exigiría un retroceso en estas tendencias. Una perspectiva no antropocéntrica cultiva el propósito humano de sanar las relaciones con la naturaleza y vivir de acuerdo con 
ella. Una forma de iniciar dicha reparación es practicar adecuadamente su restauración (Hettinger, 2002, p. 117).

Los ecocéntricos creen que existen razones intrínsecas a la naturaleza y completamente independientes de cualquier interés humano de preservar la naturaleza no humana. Para los ecologistas humanistas, sin duda hay buenas razones para preservar la naturaleza no humana, razones basadas en una responsabilidad por intereses humanos construidos por alguien. Es así que para los ecocéntricos, los argumentos de los humanistas son equivocados y posiblemente misantrópicos en su degradación moral de los intereses humanos (Humphrey, 2000, p. 249).

La ética ecocéntrica se origina del término acuñado en 1913 como ética biocéntrica (Lawrence Henderson) para representar la idea que el universo es el creador de la vida (Campbell, 1983). Este término fue adoptado por los ecologistas profundos alrededor de 1970, para referirse a la idea de que toda la vida tiene un valor intrínseco además de su utilidad para los seres humanos. La ética medioambiental o nueva ética "intenta fundamentar racionalmente los principios, valores y normas que rigen la conducta del hombre con la naturaleza, más allá del círculo de intereses morales humanos" (Lecaros, 2009b, p. 69). La ética ecocéntrica se enmarca en el aforismo o máxima de Aldo Leopold "una acción es correcta cuando tiende a preservar la integridad, la estabilidad y la belleza de la comunidad biótica, se equivoca cuando afirma lo contrario". Desde esta visión, todas las acciones humanas deben ser juzgadas como instrumentos para proteger el valor intrínseco de la comunidad biótica. Dado que Leopold fue formado bajo un enfoque utilitario centrado en los humanos para gestionar recursos, lo presenta como valores no antropocéntricos (Dietz; Fitzgerald; Shwom, 2005, p. 343).

Una característica distintiva de la ética ecocéntrica es su holismo que valora las entidades colectivas tales como las especies y ecosistemas sobre las individuales. Censura otras perspectivas éticas como el individualismo injustificable, en respuesta ha sido estigmatizado como fascismo ambiental. Los protagonistas del ecocentrismo han conceptualizado a los humanos y no humanos como miembros de una comunidad, como elementos de un gran organismo, como partes de una máquina o como aspectos de un flujo de energía (Cheyne; Alder, 2007, p. 187-188). El ecosistema, un ente holístico, tiene valor por encima del valor de sus componentes individuales, incluso en la mayoría de los casos tiene un vale más. De este modo, la caza es el principal argumento de Leopold para explicar el ecocentrismo, si bien la aprueba, rechaza los programas de erradicación de depredadores ya que empobrecen los ecosistemas. Por ejemplo, dejar pocos lobos en una montaña significa aumentar la población de ciervos, en cambio su completa ausencia representa un paraíso para los cazadores. En conclusión, la erradicación de depredadores afecta el ecosistema de la montaña y frente a una sobrepoblación de ciervos la demanda de hierba para su alimentación aumentará (Wenz, 2003, p. 107-108).

Una ética deontológica no antropocéntrica se deriva del supuesto que la mayoría de mamíferos son autónomos y por lo tanto merecen el mismo respeto que profesamos entre los humanos, sujetos a diferentes intereses, capacidades y necesidades de diferentes especies (Cheyne; Alder, 2007, p. 185).

Para los ecocéntricos, una teoría adecuada del valor intrínseco de la naturaleza, permite conocer cuáles especies naturales deben ser preservadas, cómo establecer intercambios entre las formas de vida en diferentes niveles de agregación y decidir en qué grado se pueden perseguir intereses humanos consistentes con el respeto del valor de especies naturales no humanas. Esto es resolver, por ejemplo, el conflicto entre los intereses individuales de preservación de un lobo hambriento y los intereses de su ecosistema de reducir la población de lobos, de modo que otras especies de depredadores resurgirán (Whiteside, 2004, p. 362).

En la ética ambiental han sido identificadas dos perspectivas, una utilitarista y una deontológica. La utilitarista depende de un análisis de costo/beneficio que asume conmensurabilidad entre intereses en competencia. En un nivel más amplio, refleja el conocido debate sobre la proporción en la que los valores ambientales son susceptibles a la racionalidad científica y la forma de cómo pueden ser objetivamente valorados e intercambiados. La perspectiva deontológica asume que un acto puede ser equivocado al no tener en cuenta ningún saldo de consecuencias; constituye un pilar para reconocer la posesión de derechos por parte de los no humanos, que podría aumentar la posibilidad de que un derecho humano pueda ser conceptualizado como en la competencia con los derechos de otras especies (Cheyne; Alder, 2007, p. 183-184).

A veces, el ecocentrismo es calificado como fascismo ambiental. Protagonistas del ecocentrismo han conceptualizado a individuos humanos y no humanos como miembros de una comunidad, como elementos de un gran organismo, como partes de una máquina o como aspectos de un flujo de energía. En torno a esto, Leopold recomienda respetar los procesos naturales tan sólo por razones prudentes, incluso su aforismo sugiere que las preferencias humanas pueden imponerse sobre la naturaleza, ya que la belleza argumentada no tiene sentido en ausencia de los humanos (Cheyne; Alder, 2007, p. 187).

Los filósofos ambientales no antropocéntricos han argumentado que el antropocentrismo legitima una actitud puramente instrumental hacia otros seres vivos menguando la naturaleza no humana. Los discursos morales no antropocéntricos comparten la convicción de que las ideologías políticas dominantes que han ayu- 
dado a dar forma al mundo moderno, han exaltado y celebrado la humanidad a expensas de la naturaleza no humana. La demanda ética ha sido desarrollar un nuevo vocabulario moral que reconozca la membresía humana en una comunidad moral y respete el valor de las especies no humanas por su propio bien. La demanda política y legal ha sido por desarrollar nuevas instituciones que privilegien el florecimiento mutuo del mundo humano y no humano (Eckersley, 2007, p. 305).

Teóricos de la tradición liberal han adoptado un enfoque de bienestar animal, uno de sus debates claves es determinar si los animales deben ser receptores de justicia. Rawls ha sugerido que la inferioridad moral de los animales implica que no pueden ser considerados como beneficiarios de justicia en el supuesto de que, solamente las personas morales - quienes puedan entender lo que es justo y que sean capaces de reclamar por si mismos y respetar los derechos de los demás - tienen derecho a ser beneficiarias de la justicia (Garner, 2003, p. 235). En términos kantianos, la crueldad con los animales se debe evitar, no porque sean directamente sujetos de derechos morales, sino porque la crueldad con los animales por placer enrudece la naturaleza humana y la hace menos humana (Cheyne; Alder, 2007, p. 184). Dado que la relación entre la protección animal y el liberalismo es problemática, se ha explorado un ajuste con otras tradiciones ideológicas. Una de dichas ideologías es el comunitarismo que garantiza la protección y el respeto por los animales. Adicional al comunitarismo se identifica el marxismo, el conservatismo, el feminismo y la ecología.

La diferencia entre ecocentrismo y biocentrismo ha sido claramente establecida. El ecocentrismo engloba un conjunto de éticas "que creen en el valor inherente de toda la naturaleza y consideran moral e integralmente a los ecosistemas, a la biosfera y a la Tierra". El biocentrismo refiere un conjunto de éticas "que se centran exclusivamente en la consideración moral del ser vivo". En torno al biocentrismo se han desarrollado distintos enfoques: 1) El zoocentrismo que reserva la consideración moral para especies que sienten y para individuos con conciencia; 2) El biocentrismo fuerte o igualitarista argumenta el respeto de especies animales y vegetales independiente de las capacidades; 3) El biocentrismo moderado considera el valor de la vida haciendo la salvedad que no todos los seres gozan de la misma consideración moral, pues ésta depende de la complejidad de cada sistema vivo. (Lecaros, 2009b, 63-64).

\section{Enfoques centrados en el hombre}

Antropocentrismo significa centrado en el hombre, bajo esta óptica el ser humano es un fin y la naturaleza con sus especies - animal y vegetal - es un medio; el hombre tiene deberes indirectos con la naturaleza en la medida que proporcione bienestar humano. Mientras que para el ecocentrismo, cortar un bosque tropical es malo porque puede causar la extinción de especies vegetales y animales, para el antropocentrismo es inadecuado porque puede contener curaciones potenciales para enfermedades humanas (Kortenkamp; Moore, 2001, p. 262).

Es así que los antropocéntricos sostienen que lo que hace el ser humano por la naturaleza (y por su propio bien) es suficiente, que no hay otro tipo de obligaciones para con ella. Para ellos, la naturaleza no es de interés debido a su falta de valor intrínseco y la asignación de un valor puramente instrumental (Martinelli, 2008, p. 79). El antropocentrismo débil "considera un espectro más amplio de los valores humanos en relación con la naturaleza (como los científicos, los estéticos y los espirituales), no reconoce su valor intrínseco (Lecaros, 2009a, p. 70).

Según Campbell (1983), el término antropocentrismo fue acuñado en 1860 en medio de la controversia sobre la teoría de la evolución de Darwin, para representar la idea de que los humanos son el centro del universo. El antropocentrismo considera la humana como la forma de vida más importante, otras formas lo serán sólo en la medida que impacten o puedan ser útiles a los humanos. Bajo una ética antropocéntrica, la naturaleza goza de consideración moral ya que degradarla o preservarla puede en consecuencia perjudicar o beneficiar a los humanos (Kortenkamp; Moore, 2001, p. 262).

El antropocentrismo considera no sólo la supremacía de los seres humanos, sino también, la conquista de la naturaleza y su manipulación para el beneficio exclusivo de la humanidad. Frente a esta visión, Leopold propone en el marco de la ética de la Tierra que se cambie el papel del hombre de conquistador de la misma a simple miembro y ciudadano de ella. Implica entonces respeto por los demás miembros y por la comunidad como tal (Wenz, 2003, p. 110). Según Zimmerman, en el antropocentrismo las cosas tienen valor sólo en la medida en que es útil en la promoción de los propósitos - especialmente la seguridad, comodidad y capacidad.

Al igual que Arne Naess, Schoenichen recomienda dividir la tierra en tres regiones: urbana-industrial, uso mixto y silvestre. La primera región fundamentalmente antropocéntrica, se puede entregarla para el uso intensivo de la humanidad, limitando estrictamente la contaminación para proteger las granjas, bosques y áreas silvestres de la periferia. En la región mixta, alguna intervención humana ambientalmente sostenible es permitida (silvicultura, agricultura), la planificación ambiental y estética deben ser consideradas. Las regiones silvestres deben contar con barreras y cercos de protección natural a fin de prevenir la intervención humana permitiendo excepcionalmente actividades 
no intrusivas como las caminatas o la investigación científica (Zimmerman, 1995, p. 213).

El interés antropocéntrico o antropocentrismo se refiere a la idea que los humanos son el centro del universo y que el ambiente debe ser protegido por su valor en el mantenimiento o la mejora de la calidad de vida humana. El antropocentrismo aparece como una combinación de orientaciones de valores altruistas y egoístas, los individuos son menos propensos a apoyar la protección del ambiente, pues, por lo general, esto amenaza otros valores de los humanos (Casey; Scott, 2006, p. 58). La idea del valor del antropocentrismo ha sido apoyada por autores como Thompson y Barton, para quienes el valor de la naturaleza depende del uso humano combinando autointerés y altruismo humanista.

El enfoque antropocéntrico evalúa asuntos éticos exclusivamente sobre las bases del perjuicio o beneficio a los seres humanos. A partir de una perspectiva utilitarista, la crueldad con los animales puede además llevarnos a ser crueles con otros humanos. Bajo la visión del antropocentrismo iluminado, la protección ambiental está muy relacionada con los intereses humanos, pero no se descarta que se reflejen valores emocionales e intereses culturales. Esto incluye el respeto por la naturaleza como algo externo y superior a nosotros de la cual se extrae el valor espiritual. Esta visión no coincide con el radicalismo de las perspectivas no antropocéntricas basadas en la premisa de la "ceguera de las especies", bajo la cual los intentos por distinguir a los humanos de otras especies son arbitrarios, dada la supuesta superioridad de la capacidad moral de los humanos. Por otra parte, estas perspectivas están soportadas en la igualdad de las especies (Cheyne; Alder, 2007, p. 184-185). El antropocentrismo sostiene que los humanos son el centro del universo, la cima de la evolución y los únicos seres que importan desde un punto de vista moral (Eckersley, 2007, p. 305). Se trata de un enfoque de los asuntos de contaminación, superpoblación y otras formas de degradación ecológica en términos de intereses humanos (Scriven apud Amrock, 2006, p. 598).

La ética antropocéntrica infiere que el valor de la naturaleza emerge de las actitudes que los seres humanos asumen hacia ella. Los árboles, caídas y mariposas no poseen una posición moral más allá de su capacidad para servir a los fines humanos, o quizás como depósitos de valores importantes para los humanos. En si mismos, no tienen un valor significativo o éticamente significante (DiZerega, 1995, p. 239-240). La crisis ecológica y el aumento del activismo ambiental han generado una revolución en la ética occidental que ha cuestionado lo que David Ehrenfeld (2007, p. 305) denominó "la arrogancia del humanismo" o lo que ha sido conocido como antropocentrismo o chauvinismo humano.

En 1973 fue distinguida la ecología superficial al reconocer que los recursos son agotables y que el con- tinuo progreso de la civilización requiere precaución en su uso. Se trata de un movimiento comprometido en la lucha contra la contaminación y el agotamiento de los recursos para garantizar la salud y la prosperidad de la población en los países desarrollados. Representa una posición antropocéntrica que argumenta el tratamiento responsable del ambiente sobre las bases de los beneficios materiales que acumularán los seres humanos. Se denomina superficial en su intento de reformar el sistema sin retar el paradigma dominante o el sistema social y económico del cual emerge (Kirkman, 2002, p. 20). Esta ecología surge de una visión de la naturaleza como un universo material, incluyendo los organismos humanos, una máquina que puede ser entendida en términos de sus pequeños componentes. Otra característica de esta visión fue una obsesión por la dominación y por el control. Creer que el acuerdo alcanzado por el hombre sobre la naturaleza implica su dominación, ha significado una amenaza para las especies (humanas o no). Este es un movimiento que justifica el uso de la ciencia y de la tecnología para controlar, manipular y explotar la naturaleza (Hoy, 2000, p. 93).

\section{Reflexiones Finales}

Posiblemente al ecocentrismo no se le ha dado una buena lectura, establecer límites a la actividad humana conlleva evitar su explotación, pero no su uso para la satisfacción de necesidades básicas de los humanos. El hecho que el ecocentrismo no identifique a la naturaleza como un fin ni al hombre como un medio, deja abierta la posibilidad de finalidades humanas permitiendo el uso de algunos de sus recursos. Contrariamente, el antropocentrismo al establecer el ser humano como único objeto de valor en el mundo natural o como el objeto de mayor valor, no otorga derecho ni valor a la naturaleza, significa que bajo cualquier consideración el hombre siempre resultará valioso en detrimento de los intereses de las especies no humanas. El ecocentrismo en ningún momento trata de otorgarle a la naturaleza un carácter sagrado o místico.

Cuando resulte inevitable la satisfacción de necesidades básicas del hombre, en el marco de la igualdad de las especies del biocentrismo, deberá garantizarse un mínimo de daño posible y, en caso de producirse la obligación su restitución. A su vez, no importa los motivos del antropocentrismo, ya que las actividades humanas y empresariales garantizan la preservación de la integridad y la estabilidad de la naturaleza, sus especies y recursos. Contra lo que muchos suponen, el antropocentrismo si pregona la protección y conservación de la naturaleza, sólo que con el ánimo de ase- 
gurar la salud y la prosperidad de la especie humana, a diferencia del ecocentrismo que lo hace en pro del bienestar de las especies naturales.

Desconocer o ignorar las versiones del desarrollo sostenible puede llevar a entender que privilegiar los intereses de las especies y los recursos naturales se está inclinando hacia el ecocentrismo y que promocionar los intereses de la sociedad es optar por el antropocentrismo. Las dos interpretaciones consideran mutuamente la naturaleza y el hombre, sólo que, las acciones obedecen a diferentes jerarquías de intereses. La interpretación fuerte se niega a tratar a las especies humana y natural por separado, en su lugar busca mantener las oportunidades de vida, reconciliando el desarrollo humano y la integridad ambiental.

En estos tiempos, dominados por una tecnificación industrial rampante y por un consumismo inconsciente, la ecología profunda no puede verse como una apuesta fascista o radical es más bien una salida necesaria, pues sólo así se invertirán las tendencias destructivas. Los problemas enfrentados en una región urbana-industrial en países como Colombia, los problemas no son ecológicos sino que se encuentran en la ecología, es decir, que pueden ser minados o solucionados a través de los cambios tecnológicos.

\section{Referencias}

AMROCK, Julia. Challenges for private sector conservation: Sanderson's the future of conservation in Tierra del Fuego. Indiana Journal of Global Legal Studies, v. 13, n. 2, p. 595-615, sum. 2006.

BARKIN, J. Samuel. Discounting the discount rate: ecocentrism and environmental economics. Global Environmental Politics, v. 6, n. 4, p. 56-72, nov. 2006.

CASEY, Paul J.; SCOTT Kylie. Environmental concern and behavior in an Australian sample within an ecocentric - anthropocentric framework. Australian Journal of Psychology, v. 58, n. 2, p. 57-67, sep. 2006.

CHEYNE, Ilona; ALDER John. Environmental ethic and proportionality. Hunting for a balance. Environmental Law Review,. n. 9, p. 171-189, 2007.

CURTIN, Deanne. Chinnagounder's challenge. The question of ecological citizenship. Bloomington: Indiana University Press, 1999.

DIETZ, Thomas; FITZGERALD, Amy; SHWOM Rachael. Environmental Values. Annual Review of Environment E Resources, n. 30, p. 335-372, 2005.

DIZEREGA, Gus. Empathy, society, nature and the relational self: deep ecology and liberal modernity. Social Theory and Practice, v. 21, n. 2, p. 239-269, sum. 1995.

DIZEREGA, Gus. Deep Ecology and Liberalism: The Greener Implications of Evolutionary Liberal Theory. Review of Politics, v. 58, n. 4, p. 699-734. fall 1996.
El dilema no es entre el biocentrismo y el antropocentrismo, es definir cuál de las dos versiones garantizará a los países en desarrollo y cuáles intereses serán perseguidos. La teoría permite confirmar que cualquiera de las dos apuestas sería adecuada para enfrentar las problemáticas ambientales y sociales, en un país como Colombia implicaría otorgarle a la naturaleza, en el peor de los casos, un valor instrumental y un mejor valor intrínseco.

Algo no considerado en las referencias del desarrollo sostenible es que sus versiones tienen una relación profunda con las tradiciones ideológicas. El biocentrismo con el comunitarismo, marxismo, conservatismo, feminismo y la ecología; el antropocentrismo con el individualismo liberal, utilitarismo, tecnocentrismo, egocentrismo y el mecanicismo. Desde el punto de vista de la complejidad es la ecología profunda la que considera la naturaleza como un organismo y no como un agregado de componentes, en este propósito, el policentrismo y el acentrismo toman lugar a medida que aumenta el nivel de complejidad. Las relaciones en ecología nunca son lineales, comprenden una serie de interrelaciones muy complejas, y cualquier intento por aislar o por controlar una sola actividad resulta reduccionista e incluso inapropiada (Rao, 1998, p. 65-66).

ECKERSLEY, Robyn. Ecological intervention: prospects and limits. Ethics \& International Affairs, v. 21, n. 3, p. 293316. 2007.

EDWARDS, Andres. R. Sustainability revolution: portrait of a paradigm shift. Gabriola Island: New Society Publishers, 2005. ESBJÖRN-HARGENS, Sean. Integral ecology: the what, who, and how of environmental phenomena. World Futures: The Journal of General Evolution, n. 61, p. 5-49, 2005.

FERGUS, A.; ROWNEY, J. ¿Sustainable Development: Lost Meaning and Opportunity? Journal of Business Ethics, n. 60, p. 17-27, 2005.

GARNER, Robert. Political ideologies and the moral status of animals. Journal of Political Ideologies, v. 8, n. 2, p. 233-246, 2003.

GARRARD, Greg. Ecocriticism and Education for Sustainability. Pedagogy: Critical Approaches to Teaching Literature, Language, Composition, and Culture, v. 7, n. 3, p. 359-383. 2007

HERMANS, Frans; KNIPPENBERG, Luuk. A principle-based approach for the evaluation of sustainable development. Journal of Environmental Assessment Policy and Management, v. 8, n. 3, p. 299-319, sep. 2006.

HETTINGER, Ned. The problem of finding a positive role for humans in the natural world. Ethics \& The Environment, v. 7, n. 1, p. 109-123, 2002. 
HOY, Terry. Toward a naturalistic political theory: Aristotle, Hume, Dewey, Evolutionary Biology \& Deep Ecology. Westport: Praeger, 2000.

HUMPHREY, Mathew. Nature' in deep ecology and social ecology: contesting the core. Journal of Political Ideologies, v. 5, n. 2, 247-268. 2000.

KALliO, Tomi J.; NORDBERG Piia; AHONEN Ari. Rationalizing Sustainable Development - a Critical Treatise. Sustainable Development, n. 15, p. 41-51, 2007.

KIRKMAN, Robert. Skeptical Environmentalism: the limits of philosophy and science. Bloomington: Indiana University Press, 2002.

KLOSTERMANN, Judith ; CRAMER Jacqueline. The contextual meaning of sustainable development: the case of the Dutch Drinking Water Sector. Sustainable Development, n. 14, p. 268-276, mayo 2006.

KORTENKAMP, Katherine V. ; MOORE, Colleen F. Ecocentrism and anthropocentrism: moral reasoning about ecological commons dilemmas. Journal of Environmental Psychology, v. 21, n. 3, p. 261-272, 2001.

LECAROS, Juan Alberto. El puesto del hombre en la naturaleza: el problema del antropocentrismo. Revista Selecciones de Bioética, n. 15, p. 69-74, 2009a.

LECAROS, Juan Alberto. El respeto a la vida: el biocentris- mo en la ética medio ambiental. Revista Selecciones de Bioética, n. 15, p. 63-68, 2009b.

MARTINELLI, Dario. Anthropocentrism as a social phenomenon: semiotic and ethical implications. Social Semiotics, v. 18, n. 1, p. 79-99, mar. 2008.

MATHEWS, Freya. Ecological Self. London: Routledge, 1993. PALMER, Joy A. (Ed.). Fifty Key Thinkers on the Environment. London: Routledge, 2000.

RAO, C. P. (Ed.) Globalization, privatization and free market economy. Westport: Quorum Books, 1998.

SCOTT, Peter. Political theology of nature. West Nyack: Cambridge University Press, 2003.

WENZ, Peter. S. Leopold's novel. The land ethic in Barbara Kingsolver's Prodigal Summer. Ethics \& The Environment, v. 8, n. 2, p. 106-125, 2003.

WHITESIDE, Kerry. H. Beyond the Nature - Culture Dualism: The Ecology of Earth-Homeland. World Futures, n. 60, p. 357-369, 2004.

ZIMMERMAN, Michael E. The threat of ecofascism. Social Theory and Practice, v. 21, n. 2, p. 207-269, sum. 1995.

ZIMMERMAN, Michael E. Deep Ecology, Ecoactivism and Human Evolution. Revision, v. 24, n. 4, p. 40-45, spr. 2002 .

\title{
Versions of sustainable development
}

\begin{abstract}
Within the framework of sustainable development there is a tendency to relate uniquely the emphasis on environment to biocentrism and on society to anthropocentrism, ignoring the true essence of these versions and the existence of other [intermediate] versions. The anthropocentrism is based on the fulfillment of basic interests and vital needs of human being by avoiding or reducing damage to nature. For its part, biocentrism seeks recognize the intrinsic value of species, allowing the use of nature but not its exploitation or domination. Although biocentrism is fundamentally based on ethics and anthropocentrism on politics, one cannot ignore that both versions have a broad relationship with ideological traditions.
\end{abstract}

Keywords: ecocentrism; anthropocentrism, sustainable development.

\section{Versões do desenvolvimento sustentável}

Resumo: No marco do desenvolvimento sustentável existe a tendência a relacionar o privilégio do meio ambiente com biocentrismo e o da sociedade com antropocentrismo, desconhecendo a verdadeira essência dessas versões e a existência de outras. Desde o antropocentrismo, é válida a satisfação de interesses básicos e de necessidades vitais do homem, se evitado ou reduzido o dano causado à natureza. Por outro lado, o biocentrismo procura o reconhecimento do valor intrínseco das espécies, permitindo o uso da natureza mas não a sua dominação. Ora, o biocentrismo apoia-se na ética e o antropocentrismo, na política, mas não se pode desconhecer que as duas versões têm a ver com tradições ideológicas.

Palavras-chave: ecocentrismo; antropocentrismo; desenvolvimento sustentável. 